\title{
Menstrual Blood Mesenchymal Stem Cells: Boon in Therapeutics
}

\section{K. Pushkala ${ }^{1}$ and PD Gupta ${ }^{2 *}$}

${ }^{1}$ Former, Associate Professor, SDNB Vaishnav College for Women, Chennai India.

${ }^{2}$ Former, Director Grade Scientist Centre for Cellular and Molecular Biology, Hyderabad, India.

*Corresponding Author: PD Gupta. Director Grade Scientist Centre for Cellular and Molecular Biology, Hyderabad, India.

Received date: March 09, 2021; Accepted date: April 05, 2021; Published date: April 08,2021

Citation: K. Pushkala and PD Gupta, (2021) Menstrual Blood Mesenchymal Stem Cells: Boon in Therapeutics. J, Biotechnology and Bioprocessing 2(4); DOI: 10.31579/2766-2314/032

Copyright: @2021, PD Gupta, This is an open access article distributed under the Creative Commons Attribution License, which permits unrestricted use, distribution, and reproduction in any medium, provided the original work is properly cited.

\begin{abstract}
Stem cell therapy gained momentum for the past three decades in therapeutics. Alternative strategies are indispensable for the treatment of many diseases in the present scenario due to side effects of synthetic chemicals as drugs. Mesenchymal cells of different origin have been in use with good results, though ethical issues and limited availability is a drawback. Novel menstrual blood mesenchymal stems cells prove to be a wealth out of waste is a boon in therapeutics. In this review we bring a bird's eye view of different diseases treated with menstrual blood mesenchymal stem cells with positive results. Evolution in the use of these cells more and more will be a big relief to many who suffer with side effects of drugs.
\end{abstract}

Key words: stem cells; menstrual blood mesenchymal stem cells; therapeutic efficacy

\section{Introduction}

After knowing well that almost every drug has side effect(s), researchers wanted to find a therapeutic procedure where side effects are eliminated. The cell therapy instead, injects, graft or implant compatible viable cells, which effectuates a medicinal effect without any chemical drug. In the present scenario, a flourishing research field, cell-based therapy involves modifications of the patient's own cells or cells from a donor to fight a disease and alleviate medical conditions.

\section{Stem cells Therapy}

Cells which are capable of regeneration and differentiation into a wide range of specialized cell types are used for stem cell therapy which are of the following types

- Foetal stem cells (FSCs).

- $\quad$ Adult stem cells (ASC).

- $\quad$ Pluripotent stem cells (PSC).

Stem cells (SC) are immature or undifferentiated cells with the capacity to self-renew, differentiate and repopulate a host in vivo [1]. Remarkable plasticity or potency is hierarchical ranging from totipotent (differentiating into all cell types including placenta), pluripotent (differentiating into cells of the three germ layers, but not trophoblastic cells), multipotent (differentiating into cells of more than one type but not necessarily into all the cells of a given germ layer) to unipotent (differentiating into one type of cell only, e.g. Muscle or neuron).

FSCs appear to be more primitive and have greater multi-potentiality than their adult counterparts and can be isolated during gestation from many different tissues such as blood, liver and bone marrow as well as from a variety of extra-embryonic tissues such as amniotic fluid and placenta.
They have been observed to differ on many biological aspects such as growth kinetics, morphology, immunophenotype, differentiation potential and engraftment capacity in vivo.

Adult stem cells can be found in almost all tissues such as brain, dental pulp, muscle, bone marrow, skin and pancreas with their therapeutic potential. The adult stem cell could be multipotent (e.g. haematopoietic stem cells (HSCs) giving rise to all blood cells and adherent stromal/mesenchymal stem cells (MSCs) that give rise to bone, fat, cartilage and muscle) or unipotent (e.g. progenitor cells). Adult MSCs have the problem of being difficult to extract in sufficient numbers for therapy and/or presenting restricted plasticity and limited proliferative capacity compared with FSCs cells.

Pluripotent stem cells can be derived from the inner cell mass of the preimplantation embryo (i.e. FSCs cells) or isolated from the foetal primordial germ cell pool (PGC) above the allantois (i.e. embryonic germ (EG) cells and embryonic carcinoma (EC) cells [2,3]. Induced pluripotent stem cells have unique abilities to self-renew and to recreate functional tissues. The destruction of the blastocyst or early foetus necessary for their derivation/isolation raises ethical concerns [4], although recent work has shown that FSCs cells can be derived from single blastomeres isolated using procedures similar to those routinely used for pre-implantation genetic diagnosis $[5,6]$. Stem cells can keep dividing as long as they are alive and they have two important features: they can create other stem cells and/or they can become multiple types of more specialized cells. These cells or their derivatives when transplanted or infused, promote the repair response of diseased, dysfunctional or injured tissues. Nowadays, stem cell therapy has become an effective strategy for SCI (Spinal cord injury) $[7,8]$. The most promising stem cells are neural stem cells (NSCs), because of their ability to differentiate into neural and glial cells when transplanted in the site of injury [9]. However, some drawbacks also shadowed with NSCs such as non availability of adult NSCs for 
autologous cell transplant, and ethical concerns of the foetal-derived NSCs. Clinical trials on the use of bone marrow, hematopoietic (mobilized and recovered in blood and umbilical cord blood) and mesenchymal stem cells are in the pipe line [10].

\section{Mesenchymal stem cells (MSCs):}

Within the past several years, MSCs have been explored as vehicles for both cell therapy and gene therapy. MSCs are multipotent adult stem cells which can be differentiated into multiple cell types [11].These cells can be isolated from the bone marrow, umbilical cord blood, adipose tissue, muscle, and dental pulp. Transplantation of MSCs into the injured rat spinal cord promoted tissue preservation by directly replacing the damaged cells, decreasing the cyst and injury area, stimulating axonal sprouting, producing neurotrophic factors, as well as inhibiting inflammatory cytokines. In comparison with NSCs, MSCs show a high degree of genomic stability during culture and typically do not result in tumour formation. Centre for Genomic Regulation (CRG) in Barcelona are developing a stem cell treatment for retinal degeneration by using engineered mesenchymal stem cells (from bone marrow) to have an excessive amount of Ccr5 and Cxcr6 and transplanted them into models of retinal degeneration which preserved the function of degenerating retinal tissue. Mesenchymal stem cells could offer several advantages for the treatment of eye diseases, including an ability to differentiate into retinal cells and to be grown outside the body. When the altered cells were transplanted into models of human and mouse retinal diseases, the cells migrated in high numbers to deteriorating retinal tissue and prevented the death of retinal cells [12].

\section{Menstrual blood-derived mesenchymal stem cells (MenSCs):}

Menstrual blood-derived mesenchymal stem cells (MenSCs) are boon in therapeutics and a list of diseases are treated and more are in pipeline undergoing clinical trials [13] (Fig-1). Approximately a decade ago, Meng et al. and Cui et al. discovered a novel source of MSCs from human menstrual fluid, named menstrual blood-derived stem cells (MenSCs) $[14,15]$. These SC have high rate of proliferation and possess multi lineage differentiation potency as well as its differentiation into epidermal lineage is remarkable $[16,17]$.

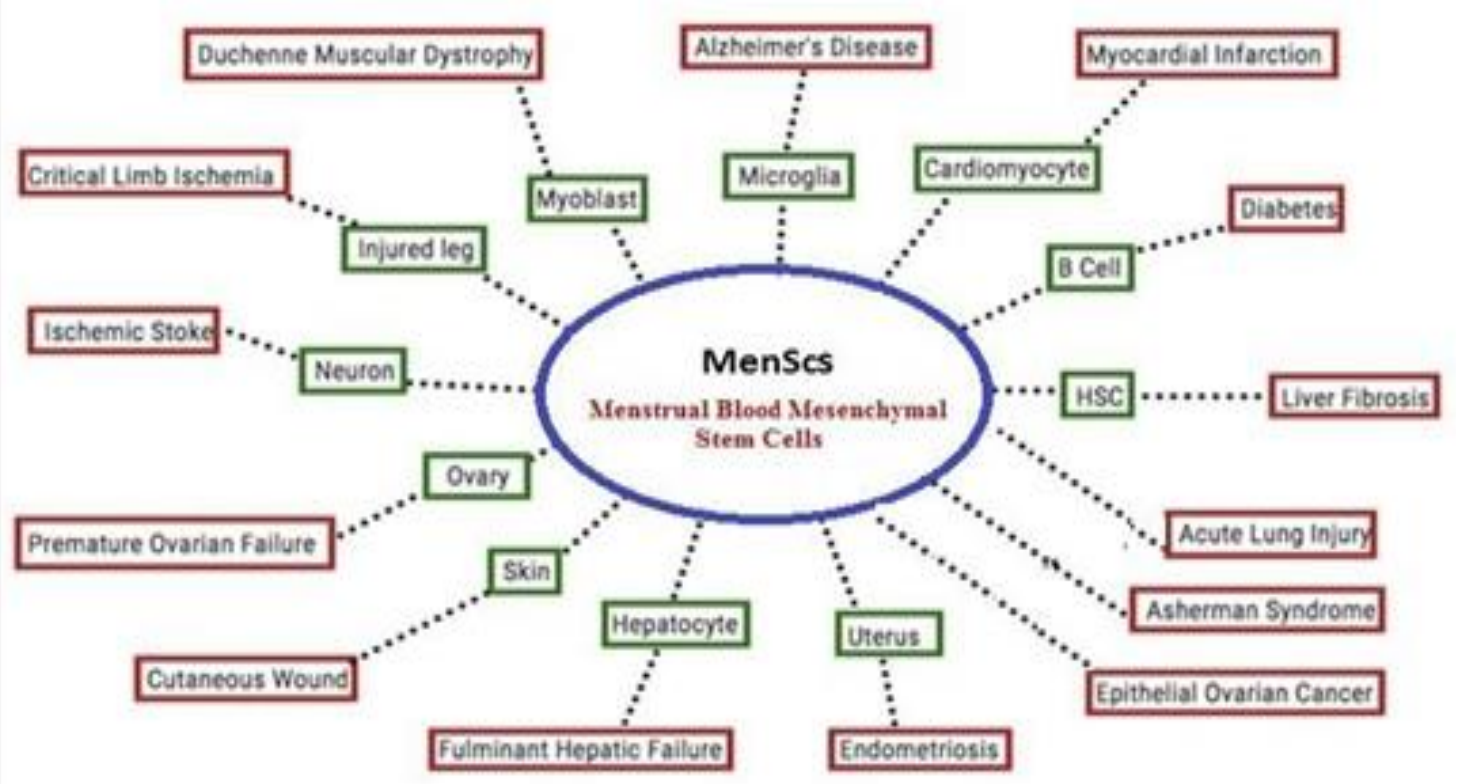

Figure 1. Different diseases treated with MenSCs.

MenSCs are giving hopes in clinical application that can be harvested from human menstrual blood shedding of endometrium which is a highly dynamic and a powerful substance. Scientists in Japan have found that cells taken from menstrual blood can be cultured in the laboratory and used like SC, which can even repair damaged heart tissue. Scientists devoted to researching SC, found that the lining of the uterus, which is a main constituent of period blood, have an abundance of these cells. MenSCs proliferate faster than the stem cells from umbilical cord and can be manipulated to transform into all kinds of cells, including those found in fat, muscle, bone, and nerves.

Menstrual fluid consists of blood, extra tissue from the uterine lining, remnants of the unfertilized egg that travelled down the fallopian tube into the uterus during ovulation. Women felt better about their periods after being made aware of the ability to donate menstrual fluid, meaning that MenSCs therapy can be beneficial for donors as well as patients. The average volume of menstrual fluid during a monthly menstrual period is
$35 \mathrm{ml}$ (2.4 table spoons of menstrual fluid), with $10-80 \mathrm{ml}$ (1-6 tablespoons of menstrual fluid) considered typical. The blood contains sodium, calcium, phosphate, iron, and chloride, the extent of which depends on the woman. In addition to blood, the fluid consists of cervical mucus, vaginal secretions, and endometrial tissue. Vaginal fluids in menses mainly contribute water, common electrolytes, organ moieties, and at least 14 proteins, including glycoproteins.

With potential for multi-directional differentiation, MenSCs are able to undergo adipogenic, chondrogenic, osteogenic, myogenic and neurogenic differentiation in vitro $[18,19]$. Compared with other sources of MSCs, MenSCs can be easily selected in a periodic manner and obtained by a noninvasive method, avoiding the ethical issues [20], do not form teratomas [21], and can be expanded by at least 20 passages without genetic abnormalities [22]. In recent studies, MenSCs showed potential therapeutic applications in a variety of animal disease models, such as stroke [23], type 1 diabetes [24, 25], and myocardial infarction [26] 
and so on. Co-culture experiments showed that MenSCs restrained the proliferation of LX-2 cells (HSC line) through secretion of paracrine cytokines, including interleukin-6 (IL- 6), interleukin-8 (IL-8), monocyte chemoattractant protein 1 (MCP-1), growth-related oncogene (GRO), osteoprotegerin (OPG), hepatocyte growth factor (HGF) by promoting hepatocellular regeneration or inducing the apoptosis of stellate cells and antifibrotic function by inducing $\mathrm{G}_{0} / \mathrm{G}_{1}$-phase arrest in stellate cells.

\section{Therapeutic applications of (MenSCs)}

\section{Uterine endometriosis}

Inactive endometrium contains hormone independent clonogenic epithelial and stromal cells which does not vary from the proliferative to secretory stage of the menstrual cycle, or between active cycling and inactive endometrium for both epithelial and stromal cells [27]. Activation of the Hippo/TAZ pathway is required for menstrual stem cells to suppress myofibroblast and inhibit transforming growth factor $\beta$ signalling in human endometrial stromal fibrosis [28].

\section{Fulminant hepatic failure}

Human menstrual blood-derived stem cells alleviate fulminant hepatic failure (FHF). Parekkadan et al. [29] for the first time have shown that - Mesenchymal Stem Cell-Derived Molecules (Exosomes) can also reverse FHF in mice in vitro and in vivo.

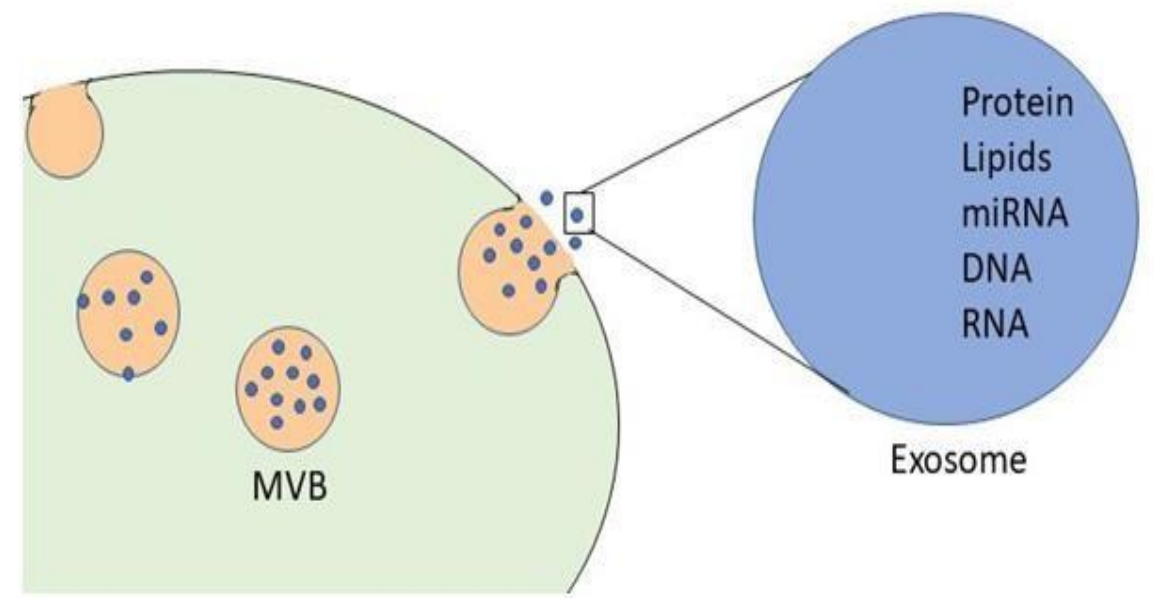

Figure 2. The origin of microvesicles and exosomes from cells

Microvesicles which arises by budding from the plasma membrane are more irregular in shape and size and can contain cytoplasmic materials. Micro vesicles express surface markers such as integrin- $\beta$, CD40 and selectins such as plasma selectins and/or proteins from the cells they originate from. Exosomes originate from the endosomal trafficking system (therefore, are more regular in shape and size), easily identifiable via cell surface markers such as CD81, CD9 and CD63 and may contain materials such as mitochondrial DNAs, mRNAs and miRNAs; microRNA; multi-vesicular bodies (MVB) (Figure 2).

\section{Skin disease}

Inflammatory skin disorders resulting in serious deterioration of the quality of life becomes one of the major public concerns. Unfortunately, despite their significance, there is no fundamental cure to date. Only BM (bone marrow) derived MSCs possess unique immunomodulatory properties which make them a promising tool for the treatment of various inflammatory diseases especially within the skin due to the suppressive effect on T cell proliferation till today. MSCs from menstrual blood can be successfully used for the treatment of atopic dermatitis (AD), dermatitis, and psoriasis. In addition, it has been proposed that cell priming or gene transduction can be novel strategies for the development of next-generation high-efficacy MSCs for treating inflammatory skin diseases [30].

\section{Premature ovarian failure (POF)}

The incidence of premature ovarian failure (POF) has been increasing in recent years. Many researchers have reported that MenMSCs, were tracked in ovarian tissues within 2 months after transplantation, and they differentiated into granulosa cells (GCs). Therefore, the use of these cells can be a practical and low-cost method for the treatment of POF patients. Several studies have shown that MenMSCs, reduce apoptosis in granulosa cells and fibrosis of the ovarian interstitium, thereby improving folliculogenesis and rescuing overall ovarian function in an animal model of POF [31], including restoring fertility [32]. In addition, Wang et al. demonstrated that MenMSCs, produced a high level of fibroblast growth factor 2, which enhanced cell survival, proliferation, and function to repair tissue damage [33], hence, we can safely conclude that the mesenchymal stem cells and in vivo survival of MenMSCs, make them ideal seed cells for stem cell transplantation in the treatment of POF.

\section{Ischemic stroke}

Transplantation of MenSCs, (either intracerebrally or intravenously and without immune suppression), after experimentally induced ischemic stroke in adult rats also significantly reduced behavioral and histological impairments compared to vehicle-infused animals. MenSCs exemplify a source of "individually tailored" donor cells that completely match the transplant recipient, at least in women. Autologous menstrual-bloodderived cells previously collected, expanded up to third passage and cryopreserved would still benefit from the use of allogeneic cells which, being stromal cells, present low immunogenicity and, therefore, tolerable rejection rates $[34,35]$.

\section{Critical Limb ischemia}

De novo angiogenesis based on MenSCs therapy is considered as a promising treatment for acute limb ischemia. MenSCs significantly improved hind limb ischemia. The recovery of the ischemic tissue returned to normal in mice. These results indicate that menstrual blood is a promising source to isolate stem cells for hind limb ischemia treatment $[36,37]$.

\section{Duchenne muscular dystrophy}

Umezawa's team $[38,39]$ successfully transdifferentiated MenSCs into myoblasts/myocyts in vitro and restored sarcolemmal expression of dystrophin in dystrophied muscle of Duchenne muscular dystrophy 
(DMD) in mice. Cellular fusion between adipocytes and dystrophied myocytes expressed dystrophin [40] providing positive results for the treatment of Duchenne muscular dystrophy.

\section{Alzheimer's disease}

Intracerebral transplantation of MenSCs dramatically improved the spatial learning and memory of APP/PS1 mice as well as significantly ameliorated amyloid plaques and reduced tau hyperphosphorylation in APP/PS1 Alzheimer's disease in mouse model. MenSCs markedly increased several A $\beta$ (amyloid-beta) degrading and anti-inflammatory impact of MenSCs in the brains of APP/PS1 mice. Similar results could be anticipated in humans also by clinical trials which are yet to be done $[13,41]$.

\section{Myocardial infarction}

Transplanted MenSCs significantly restored the damaged cardiac function, reduced apoptosis, promote cell proliferation, and stimulate angiogenesis which rescued the myocardium in the infarct region, and recruited c-kit + cells. Expression of some specific cytokines to activate AKT/extracellular signal-regulated kinases 1 and 2 (ERK 1/2)/signal transducers and activator of transcription 3 (STAT 3) and suppress p38 signaling pathway also was noticed [42, 43], Wang's team found that MenSCs inhibited endothelial cell to mesenchymal transition (EMT), which helped to reduce the total number of cardiac fibroblasts and tissue fibrosis progression [44]. The secreted exosomes of miR-21 mediated and enhanced the paracrine and cytoprotective effects through a transwell co-culture system in vitro. MenSCs transplantation decreased fibrosis area and restored the LV systolic function in the MI (myocardial infarction) model in vivo. Engrafted MenSCs are trans-differentiated into cardiomyocyte within MI area. MenSCs can be a major cell source for stem cell therapy to achieve cardiomyogenesis.

\section{Diabetes}

Intravenous injection of human menstrual blood progenitor cells (MBPCs) can reverse hyperglycemia and weight loss, prolong lifespan, and increase insulin production in diabetic mice. Histological and immunohistochemistry analysis indicated that T1DM mice with MBPCs transplantation recovered islet structures and increased the $\beta$ cell number. In vivo distribution of MBPCs when analysed a majority of MBPCs migrated into damaged pancreas and located at the islet, duct, and exocrine tissue. MBPCs did not differentiate into insulin- producing cells, but enhanced neurogenin 3 (ngn3) expression, which represented endocrine progenitors that were activated. $\mathrm{Ngn} 3^{+}$cells were not only in the ductal epithelium, but also in the islet and exocrine tissue. MenSCs could facilitate $\beta$-cell regeneration and enhance the number of $\beta$ cells by increasing the expressions of neurogenin 3 (ngn 3), forkhead box A2 (foxa 2), pancreatic and duodenal homeobox 1 ( $p d x 1)$, NK homeobox factor 6.1 (nkx 6.1), and paired box gene (pax) to activate endogenous progenitor cell differentiation in MenSCs transplantation in T1DM mice. [45].The levels of these gene expressions all increased after cell transplantation.

\section{HSC Liver Fibrosis}

Liver function was improved by MenSCs via targeting activated hepatic stellate cells (HSCs). MenSCs significantly reduced collagen deposition and improved liver function within 2 weeks of transplantation in a mouse model. These stem cells underwent hepatocyte differentiation based on CK-18 expression. Moreover, during the tracking of green fluorescent protein-expressing MenSCs demonstrated that transplanted cells migrated to the sites of injury, but few differentiated into functional hepatocyte-like cells. Results provide preliminary evidence for the antifibrotic capacity of MenSCs in liver fibrosis [46, 47, and 48].

\section{Acute lung injury}

MenSCs promoted the repair of injured lung by inhibiting the inflammatory response in LPS (lipopolysaccharide)-induced ALI acute lung injury in mice [49]. Furthermore, MenSCs not only improved pulmonary microvascular permeability, reduced histopathological injury, down regulated the expressions of IL- $1 \beta$ and caspase- 3 , up regulated the levels of IL-10 in bronchoalveolar lavage fluid (BALF) in the damaged lung, proliferating cell nuclear antigen (PCNA), and keratinocyte growth factor (KGF) in bronchoalveolar lavage fluid (BALF). MenSCs could also increase the survival rate of BEAS-2B cells (human normal lung epithelial cells) [50].

\section{Asherman syndrome}

Asherman's syndrome is an uncommon, acquired, gynaecological disorder characterized by changes in the menstrual cycle caused by the formation of adhesions in the uterine cavity. Patients experience reduced menstrual flow, increased cramping and abdominal pain, eventual cessation of menstrual cycles (amenorrhea), and, in many instances, infertility, (including amenorrhea, less menstruation, or dysmenorrhea), and repeated pregnancy loss [51]. This can be treated by autologous MenSCs transplantation that significantly increased endometrial thickness (ET) in Asherman syndrome women in 7 patients [52]. They showed that the ET of 5 women was significantly increased to $7 \mathrm{~mm}$ (a thickness to ensure embryo implantation). Four of these patients were subjected to frozen embryo transfer (FET). Surprisingly, one patient developed a spontaneous pregnancy only after the second MenSCs transplant.

\section{Epithelial ovarian cancer}

Epithelial ovarian cancer develops from the cells that cover the outer surface of the ovary. Most epithelial ovarian tumors are benign (noncancerous). EOC has been found to be advanced in most cases, with a combination of extensive abdominal metastasis, high recurrence, and chemo resistance [53]. This benign tumour can be treated successfully with MenSCs due to its intrinsic anti-tumor properties. EOC cells in vivo and in vitro provide a strategy for MenSCs -based anti-cancer therapy against epithelial ovarian cancer [54-56].

\section{Conclusion}

To conclude more research and clinical trials with menstrual blood mesenchymal stem cells therapy will pave way to solve many problems in treating patients. Development of sophisticated culturing and harvesting technologies to genetically engineer need based stem cells will be a breakthrough MenSCs in therapeutics.

\section{References}

1. Weissman I, Anderson D, Gage F. (2001) Stem and progenitor cells: origins, phenotypes, lineage commitments, and trans differentiations. Annu. Rev. Cell. Dev. Biol. 17: 387-403.

2. Thomson JA, Itskovitz-Eldor J, Shapiro SS, Waknitz M A, Swiergiel JJ, et al. (1998) Embryonic stem cell lines derived from human blastocysts. Science. 282, 1145-1147.

3. Andrews PW, Matin M M, Bahrami AR, Damjanov I, Gokhale P, Draper J S. (2005) Embryonic stem (ES) cells and embryonal carcinoma (EC) cells: opposite sides of the same coin. Biochem. Soc. Trans. 33: 1526-1530.

4. Lo B, Parham L. (2009) Ethical issues in stem cell research. Endocr. Rev. 30: 204-213.

5. Klimanskaya, I., Chung, Y., Becker, S., Lu, S. J. \& Lanza, R. (2007) Derivation of human embryonic stem cells from single blastomeres. Nat. Protoc. 2, 1963-1972.

6. Abdulrazzak H, Moschidou D, Jones G, Pascale V. Guillot PV. (2010) Biological characteristics of stem cells from foetal, cord blood and extraembryonic issues. J. R. Soc. Interface. 7: S689- 
S706.

7. Sahni V, Kessler JA. (2010) Stem cell therapies forspinal cord injury. Nat Rev Neurol. 6:363-372.

8. Mothe AJ, Tator CH. (2012) Advances in stem cell therapy for spinal cord injury. J Clin Invest. 122: 3824-3834.

9. Khoury M, Alcayaga-Miranda F, Illanes SE, Figueroa FE. (2014) The promising potential of menstrual stem cells for antenatal diagnosis and cell therapy. Front. Immunol. 5:205.

10. Trounson A, Thakar RG, Geoff Lomax G, Gibbons D. (2011) Clinical trials for stem cell therapies. BMC Medicine. 9:52.

11. Ullah I, Subbarao RB, Rho GJ. (2015) Human mesenchymal stem cells - current trends and future prospective. Biosci Rep. 35: e00191.

12. Restoring eyesight with genetically engineered stem cells ... www.fiercebiotech.com > research > restoring-eyesight-Dec 1, 2020 - Scientists who study eye diseases have long known that stem cells have properties that could help retinal cells repair themselves. They release .

13. Chen L, Qu J, Xiang C. (2019) The multi-functional roles of menstrual blood-derived stemcells in regenerative medicine. Stem Cell Res Ther. 10; 1.

14. Meng X, Ichim TE, Zhong J, Rogers A, Yin Z, Jackson J, et al. (2007) Endometrial regenerative cells: A novel stem cell population. J Transl Med. 5: 57-66.

15. Cui CH, Uyama T, Miyado K, et al. (2007) Menstrual bloodderived cells confer human dystrophin expression in the murine model of Duchenne muscular dystrophy via cell fusion and myogenic transdifferentiation. Mol Biol Cell. 18:1586-94.

16. Gupta PD. (2020) Menstrual blood: Don't waste, treasure it. J. Cell Tissue Research. 20; 2: PV 1.

17. Gupta PD, Pushkala K. (2020) Stem Cells from Menstrual Blood: Wealth Out of Waste. Stem Cells from Menstrual Blood: Wealth Out of Waste. Global J Surg Surgical Tech. 2: 1009.

18. Patel AN, Patel AN, Park E, Kuzman M, Benetti F, Francisco J et al. (2008) Multipotent menstrual blood stromal stem cells: isolation, characterization, and differentiation. Cell Transplant. 17:303-311.

19. Patel AN, Silva F. (2008) Menstrual blood stromal cells: the potential for regenerative medicine. Regen. Med. 3:443-444.

20. Hida N, Nishiyama N, Miyoshi S, Kira S, Segawa K, Uyama T, et al. (2008) Novel cardiac precursor-like cells from human menstrual blood-derived mesenchymal cells. Stem Cells (Dayt., Ohio). 26: 1695-1704.

21. Alfano AL, Candia AN, Cuneo N, Guttlein LN, Soderini A, Rotondaro C, et al. (2017) Oncolytic adenovirus-loaded menstrual blood stem cells overcome the blockade of viral activity exerted by ovarian cancer ascites. Mol. Ther. Oncolytics. 6:31-44.

22. Khoury M, Alcayaga-Miranda F, Illanes SE, Figueroa FE. (2014) The promising potential of menstrual stem cells for antenatal diagnosis and cell therapy. Front. Immunol. 5:205.

23. Borlongan CV, Kaneko Y, Maki M, Yu S, Ali M, Allickson JG, et al. (2010) Menstrual blood cells display stem cell-like phenotypic markers and exert neuroprotection following transplantation in experimental stroke. Stem Cells Dev. 19: 439452.

24. Wu X, Luo Y, Chen J, Pan R, Xiang B, Du X, et al. (2014) Transplantation of human menstrual blood progenitor cells improves hyperglycemia by promoting endogenous progenitor differentiation in type 1 diabetic mice. Stem Cells Dev. 23: 12451257.

25. Santamaria X, Massasa EE, Feng Y, Wolff E, Taylor HS. (2011) Derivation of insulin producing cells from human endometrial stromal stem cells and use in the treatment of murine diabetes. Mol Ther. 19: 2065-2071.
26. Zhang Z,Wang JA, Xu Y, et al. (2013) Menstrual blood derived mesenchymal cells ameliorate cardiac fibrosis via inhibition of endothelial to mesenchymal transition in myocardial infarction. Int. J. Cardiol. 168:1711-1714.

27. McLennan CE, Rydell AH. (1956) Extent of endometrial shedding during normal menstruation. Obstet Gynecol. 26: 605621.

28. Schwab, KJ, Chan RWS, Gargett CV, et al. (2005) Putative stem cell activity of human endometrial epithelial and stromal cells during the menstrual cycle. Reproductive biology. 84: 211241130 .

29. Parekkadan B, van Poll D, Suganuma K, et al. (2007) Mesenchymal Stem Cell-Derived Molecules Reverse Fulminant Hepatic Failure. PLoS ONE. 2: e941.

30. Shin TH, Kim HS, et al. (2017) Mesenchymal Stem Cell Therapy for Inflammatory Skin Diseases: Clinical Potential and Mode of Action. Int J Mol Sci. 18: 244.

31. Manshadi M D, Navid S, Hoshino Y, et al. (2019) The effects of human menstrual blood stem cells-derived granulosa cells on ovarian follicle formation in a rat model of premature ovarian failure. Microscopy Research and Technique. 82:635-642.

32. Lai D, Wang F, et al. (2015) Human endometrial mesenchymal stem cells restore ovarian function through improving the renewal of germline stem cells in a mouse model of premature ovarian failure. Journal of Translational Medicine.

33. Wang Z, Wang Y, et al. (2017) Study of the reparative effects of menstrual-derived stem cells on premature ovarian failure in mice. Stem Cell Research \& Therapy. 8:11-14.

34. Borlongan, CV, Kaneko Y, et al. (2010) Menstrual Blood Cells Display Stem Cell-Like Phenotypic Markers and Exert Neuroprotection Following Transplantation in Experimental Stroke. Stem Cell Devia. 19: 439-451.

35. Rodrigues MCO, Dmitriev D, et al. (2012) Menstrual blood transplantation for ischemic stroke: Therapeutic mechanisms and practical issues. Interv Med Appl Sci. 4: 59-68.

36. Vu NB, Trinh VNL, et al. (2014) Human Menstrual BloodDerived Stem Cell Transplantation for Acute Hind Limb Ischemia Treatment in Mouse Models. Regenerative Medicine .2014; 205-215 IN: Bhattacharya N, Stubblefield PG. (eds.), Regenerative Medicine: Using Non- Fetal Sources of Stem Cells, 205.

37. Anjan Das. (2008) Stem cell therapy for critical limb ischaemia A review. Indian Journal of Surgery. 71:177-81.

38. Cui CH, Uyama T, et al. (2007) Menstrual blood-derived cells confer human dystrophin expression in the murine model of Duchenne muscular dystrophy via cell fusion and myogenic transdifferentiation. Mol Biol Cell. 18: 1586-94.

39. Rodriguez AM, Pisani D, Dechesne CA, et al. (2005) Transplantation of a multipotent cell population from human adipose tissue induces dystrophin expression in the immunocompetent mdx mouse. J Exp Med. 201:1397-405.

40. Toyoda M, Ch C, Umezawa A. (2007) Myogenic transdifferentiation of menstrual blood-derived cells. Acta Myol. 26:176-8.

41. Zhao Y, Chen X, Wu Y, et al. (2018) Transplantation of human menstrual blood-derived mesenchymal stem cells alleviates Alzheimer's disease-like pathology in APP/PS1 transgenic mice. Front Mol Neurosci. 11:140.

42. Jiang Z, Hu X, Yu H, et al. (2013) Human endometrial stem cells confer enhanced myocardial salvage and regeneration by paracrine mechanisms. J Cell Mol Med. 17:1247-60.

43. Hida N, Nishiyama N, Miyoshi S, et al. (2008) Novel cardiac precursor-like cells from human menstrual blood-derived mesenchymal cells. Stem Cells. 26:1695-704. 
44. Zhang Z, Wang JA, Xu Y, et al. (2013) Menstrual blood derived mesenchymal cells ameliorate cardiac fibrosis via inhibition of endothelial to mesenchymal transition in myocardial infarction. Int J Cardiol. 168:1711-4.

45. Wu X, Luo Y, Chen J, et al. (2014) Transplantation of human menstrual blood progenitor cells improves hyperglycemia by promoting endogenous progenitor differentiation in type 1 diabetic mice. Stem Cells Dev. 23:1245-57.

46. Wang PP, Xie DY, Liang XJ et al. (2012) HGF and direct mesenchymal stem cells contact synergize to inhibit hepatic stellate cells activation through TLR4/NF-kB pathway.PLoS One. 7:e43408.

47. Parekkadan B, van Poll D, Megeed Z et al. (2007) Immunomodulation of activated hepatic stellate cells by mesenchymal stem cells. Biochem Biophys Res Commun. 363:247-252.

48. Kim MD, Kim SS, Cha HY et al. (2014) Therapeutic effect of hepatocyte growthfactor-secreting mesenchymal stem cells in a rat model of liver fibrosis. Exp Mol Med.46:e110.

49. Xiang B, Chen L, Wang X, et al. (2017) Transplantation of menstrual blood-derived mesenchymal stem cells promotes the repair of LPS-induced acute lung injury. Int J Mol Sci. 18:689.

50. Xiang B, Lu Chen L, Wang X, et al. (2017)-Transplantation of Menstrual Blood-Derived Mesenchymal Stem Cells Promotes the Repair of LPS-Induced Acute Lung Injury. Int. J. Mol. Sci. 18: 689.

51. Myers EM, Hurst BS. (2012) Comprehensive management of severe Asherman syndrome and amenorrhea. Fertil Steril. 97:160-4.

52. Tan J, Li P, Wang Q, et al. (2016) Autologous menstrual bloodderived stromal cells transplantation for severe Asherman's syndrome. Hum Reprod. 31:2723-9.

53. Kurman RJ, Shih IM. (2010) The origin and pathogenesis of epithelial ovarian cancer: a proposed unifying theory. Am J Surg Pathol. 34:433-43.

54. Lai D, Wang F, Yao X, et al. (2015) Human endometrial mesenchymal stem cells restore ovarian function through improving the renewal of germline stem cells in a mouse model of premature ovarian failure. J Transl Med. 13:155.

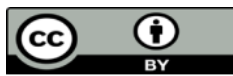

This work is licensed under Creative Commons Attribution 4.0 License

\section{To Submit Your Article Click Here: Submit Manuscript}

DOI: $10.31579 / 2766-2314 / 032$
Ready to submit your research? Choose Auctores and benefit from:

* fast, convenient online submission

* rigorous peer review by experienced research in your field

* rapid publication on acceptance

* authors retain copyrights

* unique DOI for all articles

* immediate, unrestricted online access

At Auctores, research is always in progress.

Learn more www.auctoresonline.org/journals/biotechnology-andbioprocessing 